Supporting Information

\title{
Wavelength-Turnable Organic Micro-ring Laser Arrays from Thermally Activated Delayed Fluorescent Emitters
}

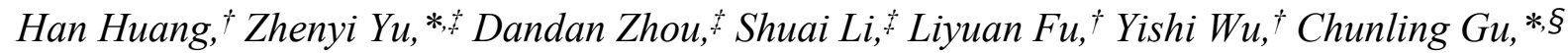
Qing Liao, ${ }^{\dagger}$ and Hongbing $F u^{*,+,+}$

${ }^{\dagger}$ Beijing Key Laboratory for Optical Materials and Photonic Devices, Department of Chemistry, Capital Normal University, Beijing 100048, P. R. China

$\$$ Tianjin Key Laboratory of Molecular Optoelectronic Sciences, Department of Chemistry, Tianjin University, and Collaborative Innovation Center of Chemical Science and Engineering, Tianjin 300072, P. R. China

$\S$ Department of Chemistry, Northwestern University

*Corresponding author: zhenyi.yu@tju.edu.cn (Z. Yu), Chunling.gu@northwestern.edu (C. Gu), hbfu@cnu.edu.cn (H. Fu)

KEYWORDS: thermally activated delayed fluorescence (TADF), micro-ring laser arrays, whispering-gallery mode, host-guest doping, wavelength tunability 
Note S1. Synthesis Procedure of the borondifluoride curcuminoid derivative (CAZ-A).

In a $50 \mathrm{~mL}$ flask, the mixture of ethyl diacetoacetate $(228 \mu \mathrm{L}, 1.463 \mathrm{mmol}, 1 \mathrm{eq})$ and $\mathrm{BF}_{3} \cdot \mathrm{Et}_{2} \mathrm{O}$ $(199 \mu \mathrm{L}, 1.609 \mathrm{mmol}, 1.1 \mathrm{eq})$ in $3 \mathrm{~mL}$ ethyl acetate was heated for 30 minutes at $50-60^{\circ} \mathrm{C}$ in air. Dissolved 9-ethyl-9H-carbazole-3-carbaldehyde (0.816 g, $3.658 \mathrm{mmol}, 2.5 \mathrm{eq})$ and $\mathrm{B}(\mathrm{n}-\mathrm{OBu})_{3}$ $(0.987 \mathrm{~mL}, 3.658 \mathrm{mmol}, 2.5 \mathrm{eq})$ into $12 \mathrm{~mL}$ ethyl acetate, then the solution was injected into the first mixture. The reaction was kept at $50-60{ }^{\circ} \mathrm{C}$ for another 30 minutes. First portion of $\mathrm{BuNH}_{2}$ $(58 \mu \mathrm{L}, 0.585 \mathrm{mmol}, 0.4 \mathrm{eq})$ was added dropwise into the reaction. After 6 hours heating, second portion of $\mathrm{BuNH}_{2}(29 \mu \mathrm{L}, 0.293 \mathrm{mmol}, 0.2 \mathrm{eq})$ was added, and the reaction was kept heating at 50-60 ${ }^{\circ} \mathrm{C}$ overnight. All the solvents were evaporated. The crude product was obtained by flash column chromatography ( silica, $\mathrm{CH}_{2} \mathrm{Cl}_{2}$ : Petroleum ether $=3: 1$ ). The further purification was done by multiple precipitations in $\mathrm{CH}_{2} \mathrm{Cl}_{2}$ /petroleum ether mixtures, giving a dark green powder (725 mg, 63\% yield). 
${ }^{1} \mathrm{H}$ NMR (600 MHz, Methylene Chloride- $d_{2}$ ) of the compound CAZ-A. $\delta 8.44-8.37(\mathrm{~m}, 4 \mathrm{H})$, $8.16(\mathrm{~d}, J=7.7 \mathrm{~Hz}, 2 \mathrm{H}), 7.82(\mathrm{dd}, J=8.6,1.7 \mathrm{~Hz}, 2 \mathrm{H}), 7.54(\mathrm{ddd}, J=8.1,6.9,1.2 \mathrm{~Hz}, 2 \mathrm{H}), 7.49$ - $7.46(\mathrm{~m}, 4 \mathrm{H}), 7.41(\mathrm{~d}, J=15.1 \mathrm{~Hz}, 2 \mathrm{H}), 7.36-7.30(\mathrm{~m}, 2 \mathrm{H}), 4.59$ (q, $J=7.1 \mathrm{~Hz}, 2 \mathrm{H}), 4.40$ (q, $J=7.3 \mathrm{~Hz}, 4 \mathrm{H}), 1.58(\mathrm{t}, J=7.2 \mathrm{~Hz}, 3 \mathrm{H}), 1.46(\mathrm{t}, J=7.3 \mathrm{~Hz}, 6 \mathrm{H})$.

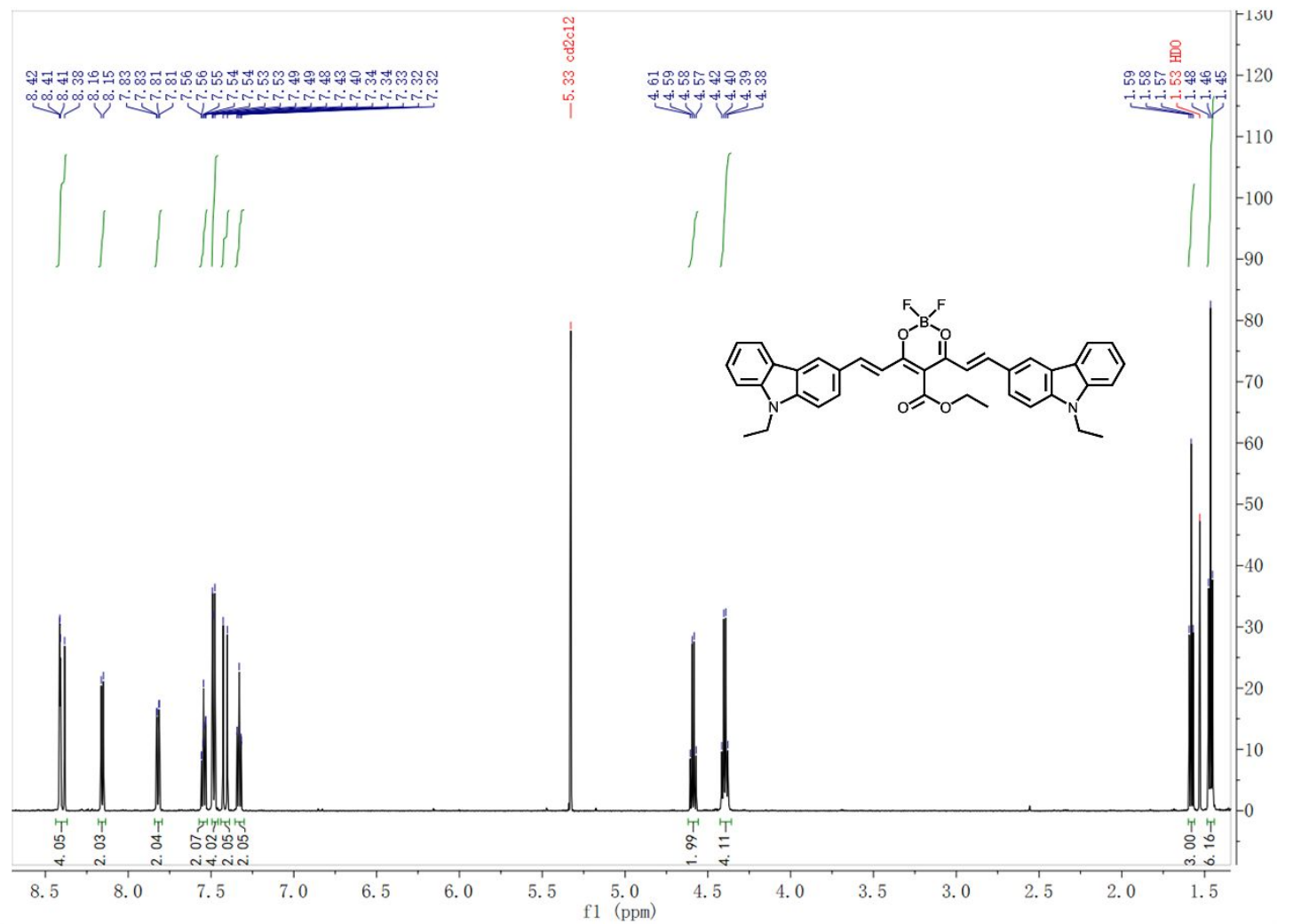

$\begin{array}{llllll}\text { Figure } & \text { S1. } & { }^{1} \mathrm{H} & \text { NMR } & \text { spectrum } & \text { of }\end{array}$ 4,6-bis((E)-9-ethyl-3-vinyl-9H-carbazole)-5-(ethoxycarbonyl)-2,2-difluoro-2H-1,3,2-dioxaborini n-1-ium-2-uide in deuterated methylene chloride. 


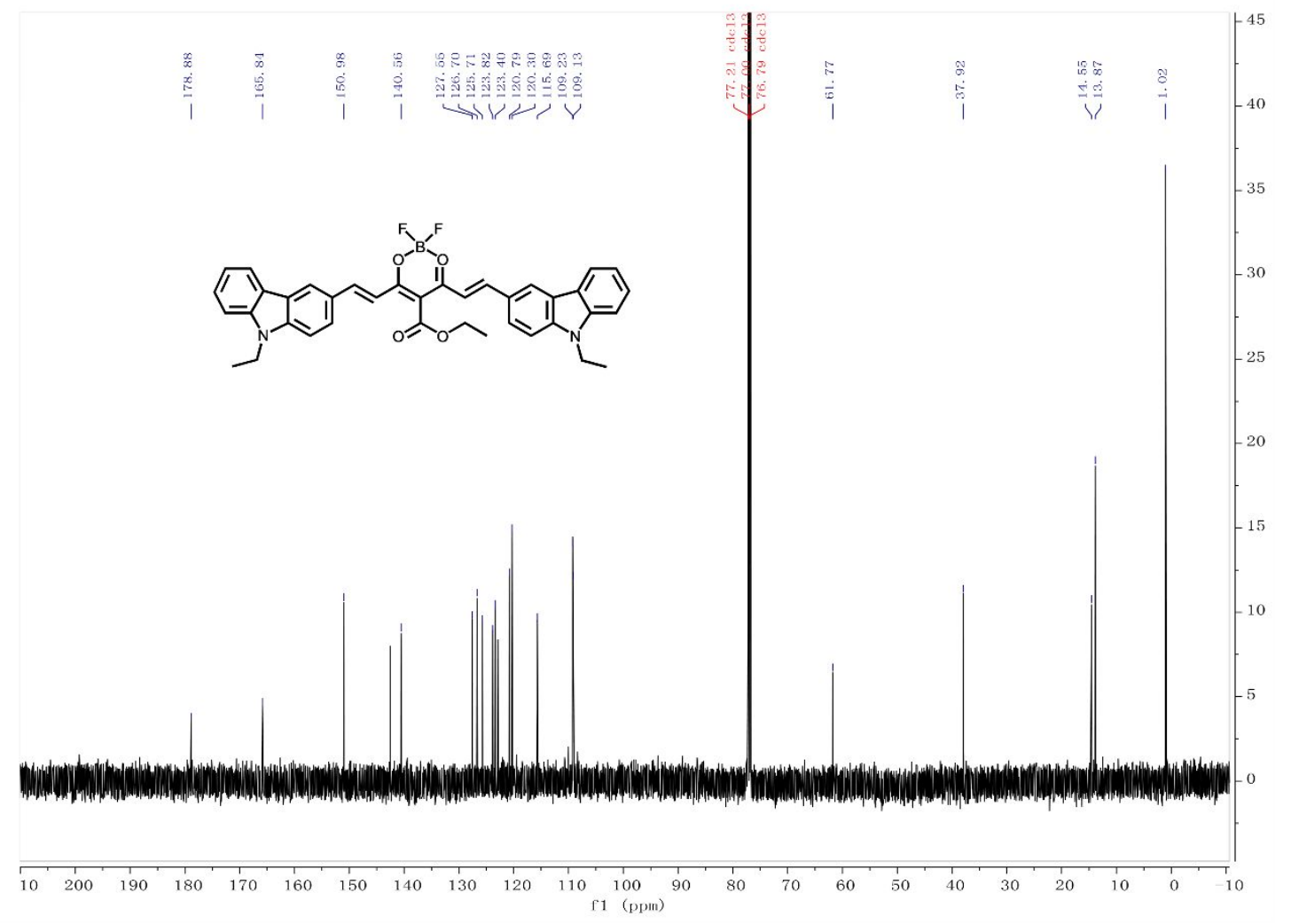

$\begin{array}{lllll}\text { Figure } & \text { S2. } & { }^{13} \mathrm{C} & \text { NMR } & \text { spectrum }\end{array}$

4,6-bis((E)-9-ethyl-3-vinyl-9H-carbazole)-5-(ethoxycarbonyl)-2,2-difluoro-2H-1,3,2-dioxaborini n-1-ium-2-uide in deuterated chloroform. 


\section{Note S2. Details on fabrication of the micro-ring-structured silicon mold and the micro-ring-structured PDMS stamp.}

Firstly, the micro-ring photomask with different sizes $(12 \mu \mathrm{m}, 20 \mu \mathrm{m}$, and $30 \mu \mathrm{m})$ and same ring gaps $(10 \mu \mathrm{m})$ was designed. A piece of $<100>$-oriented, N-doped silicon wafer with diameter of $10 \mathrm{~cm}$ and thickness of $525 \mu \mathrm{m}$ was coated with a layer of photoresist (Shipley Microposit S1800 series). Then a direct laser-writing apparatus (Heidelberg DWL200) was used to transfer the photomask patterns onto the silicon wafer with about $1 \mu \mathrm{m}$ precision. After laser irradiation and development, we used the deep reactive-ion etching technology (DRIE, Alcatel 601E) with fluorine-based reagents to fabricate the micro-ring structures. After reacting for 6 minutes, the micro-ring patterns on the wafer were fabricated successfully. Finally, we applied the regent (Microposit Remover 1165) to perform resist stripping, and used ethanol and acetone to clean the silicon mold.

Here, $20 \mu \mathrm{L}$ PDMS prepolymer and $2 \mu \mathrm{L}$ curing agent, keeping a volume ratio (10:1), were added into a $100 \mu \mathrm{L}$ breaker. They were stirred with a speed of $550 \mathrm{r} / \mathrm{min}$ for 2 hours under room temperature. Then the compound was poured into a culture dish, which included 3 different silicon molds. After standing for 20 minutes, the culture dish was placed into a vacuum drying oven. Before heating, we further distilled the residue air in the compound. Then the oven was heated to $65^{\circ} \mathrm{C}$ at constant pressure, to start the solidification process. After heating for 270 minutes, the PDMS stamp was obtained. 

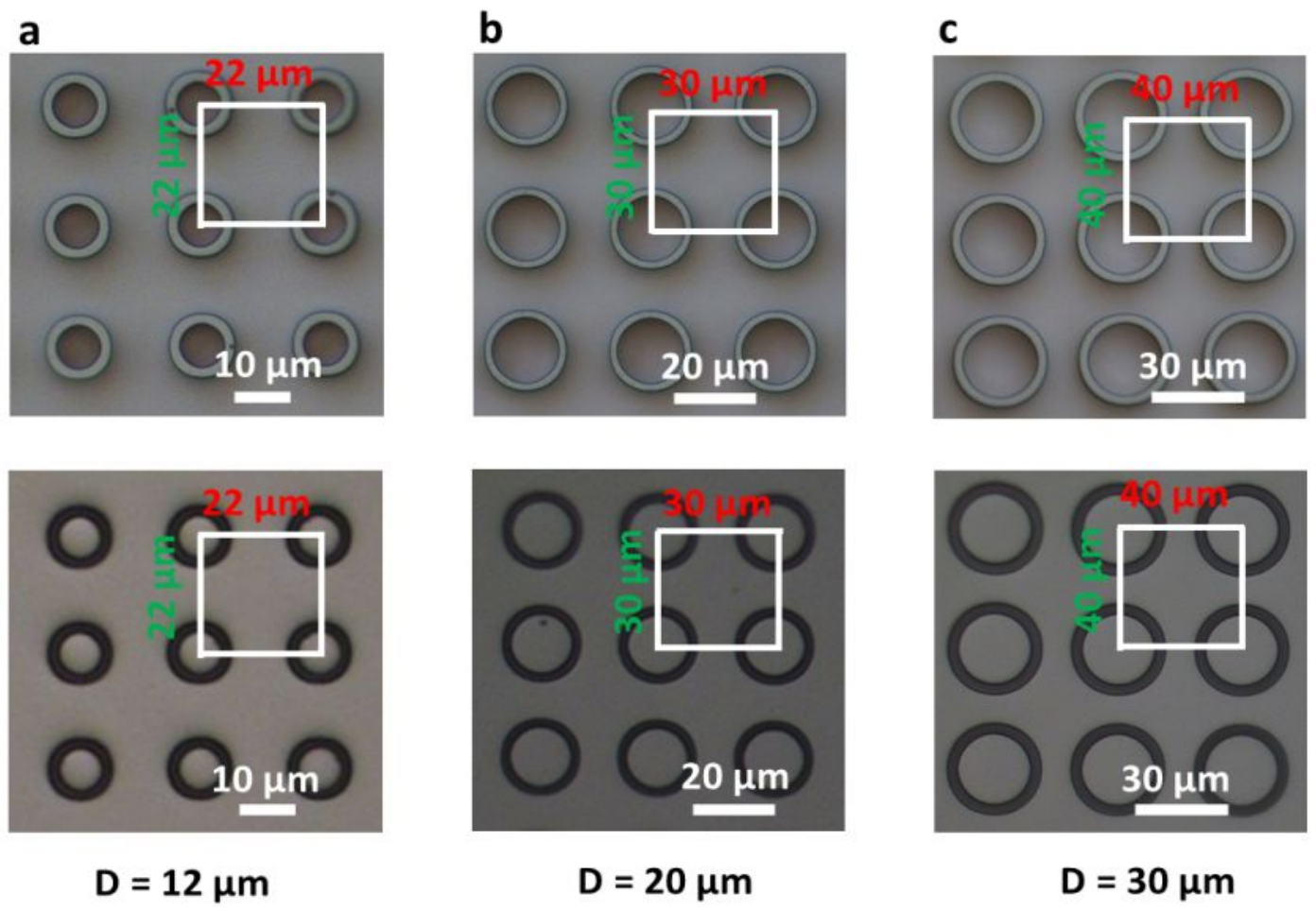

$D=20 \mu \mathrm{m}$

$$
D=30 \mu \mathrm{m}
$$

Figure S3. The optical images of three different silicon molds and PDMS stamps. a) The optical images of the silicon mold with diameter of $12 \mu \mathrm{m}$ (top) and the corresponding PDMS stamp (down). b) The optical images of the silicon mold with diameter of $20 \mu \mathrm{m}$ (top) and the corresponding PDMS stamp (down). c) The optical images of the silicon mold with diameter of $30 \mu \mathrm{m}$ (top) and the corresponding PDMS stamp (down). 
a

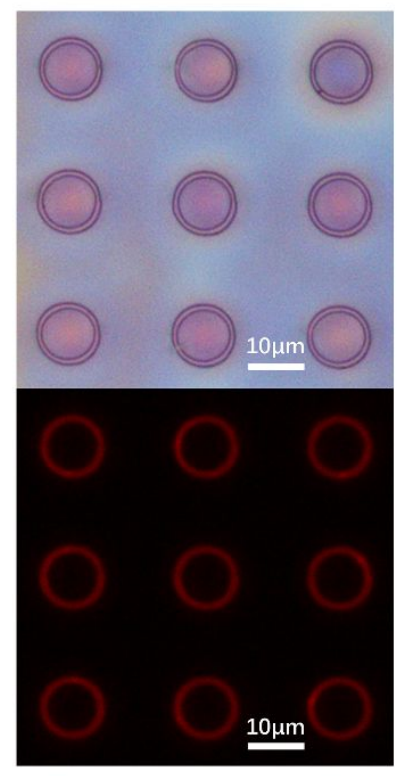

$D=11.5 \mu \mathrm{m}$ b

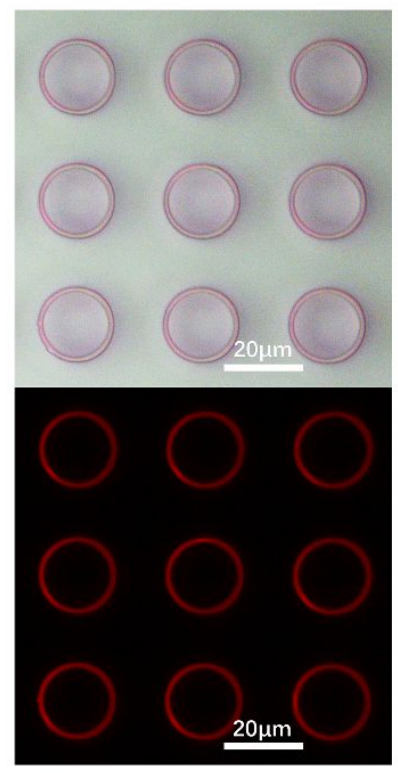

$D=19 \mu \mathrm{m}$
C
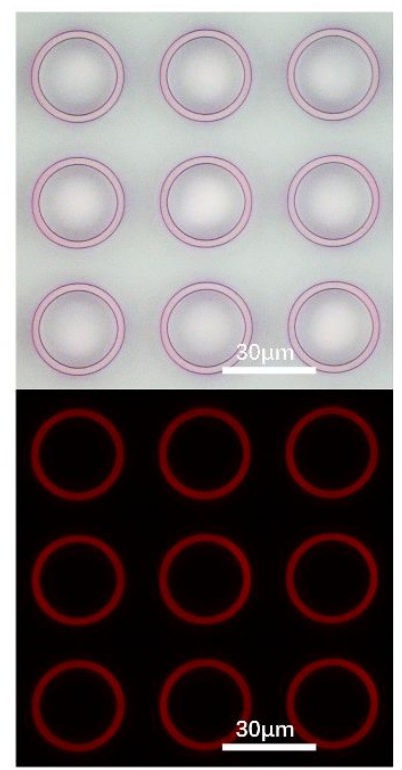

$D=29 \mu \mathrm{m}$

Figure S4. The optical and PL images of $4 \mathrm{wt} \%$ CAZ-A/CBP MRs of three different sizes. a) The optical images of the MRs with the diameter of $12 \mu \mathrm{m}$ (top) and the corresponding PL image (down). b) The optical images of the MRs with the diameter of $20 \mu \mathrm{m}$ (top) and the corresponding PL image (down). c) The optical images of the MRs with the diameter of $30 \mu \mathrm{m}$ (top) and the corresponding PL image (down). 
Table S1. Photophysical properties of CAZ-A/CBP MRs.

\begin{tabular}{|c|c|c|c|c|c|c|}
\hline \multicolumn{7}{|c|}{ CAZ-A/CBP MRs } \\
\hline Concentrations & $\begin{array}{l}\lambda_{\mathrm{em}} \\
(\mathrm{nm})\end{array}$ & $\Phi_{\mathrm{em}}$ & $\Phi_{\text {prompt }}$ & $\Phi_{\text {delayed }}$ & $\begin{array}{l}\lambda_{\text {Laser }} \\
(\mathrm{nm})\end{array}$ & $\begin{array}{c}P_{\text {th }} \\
\left(\mu \mathrm{J} / \mathrm{cm}^{2}\right)\end{array}$ \\
\hline $0.1 w t \%$ & 612 & 0.55 & & & & \\
\hline $0.3 \mathrm{wt} \%$ & 619 & 0.61 & & & & \\
\hline $0.5 w t \%$ & 637 & 0.44 & 0.36 & 0.08 & $651 \sim 668$ & 65 \\
\hline $1 w t \%$ & 641 & 0.34 & 0.281 & 0.059 & $667 \sim 676$ & 24 \\
\hline $2 w t \%$ & 644 & 0.33 & 0.288 & 0.042 & $672 \sim 681$ & 9.1 \\
\hline $4 w t \%$ & 648 & 0.17 & 0.149 & 0.021 & $677 \sim 700$ & 4.0 \\
\hline $6 w t \%$ & 653 & 0.13 & 0.114 & 0.016 & $697 \sim 706$ & 5.2 \\
\hline $8 w t \%$ & 658 & 0.13 & 0.115 & 0.015 & $695 \sim 717$ & 7.3 \\
\hline $10 \mathrm{wt} \%$ & 660 & 0.12 & 0.109 & 0.012 & $697 \sim 724$ & 8.2 \\
\hline $20 w t \%$ & 671 & 0.05 & 0.045 & 0.005 & $704 \sim 727$ & 36.1 \\
\hline $40 w t \%$ & & 0.03 & & & & \\
\hline $60 \mathrm{wt} \%$ & & 0.02 & & & & \\
\hline $\begin{array}{c}100 \mathrm{wt} \% \\
\text { ( Neat CAZ-A ) }\end{array}$ & 698 & 0.02 & & & & \\
\hline
\end{tabular}



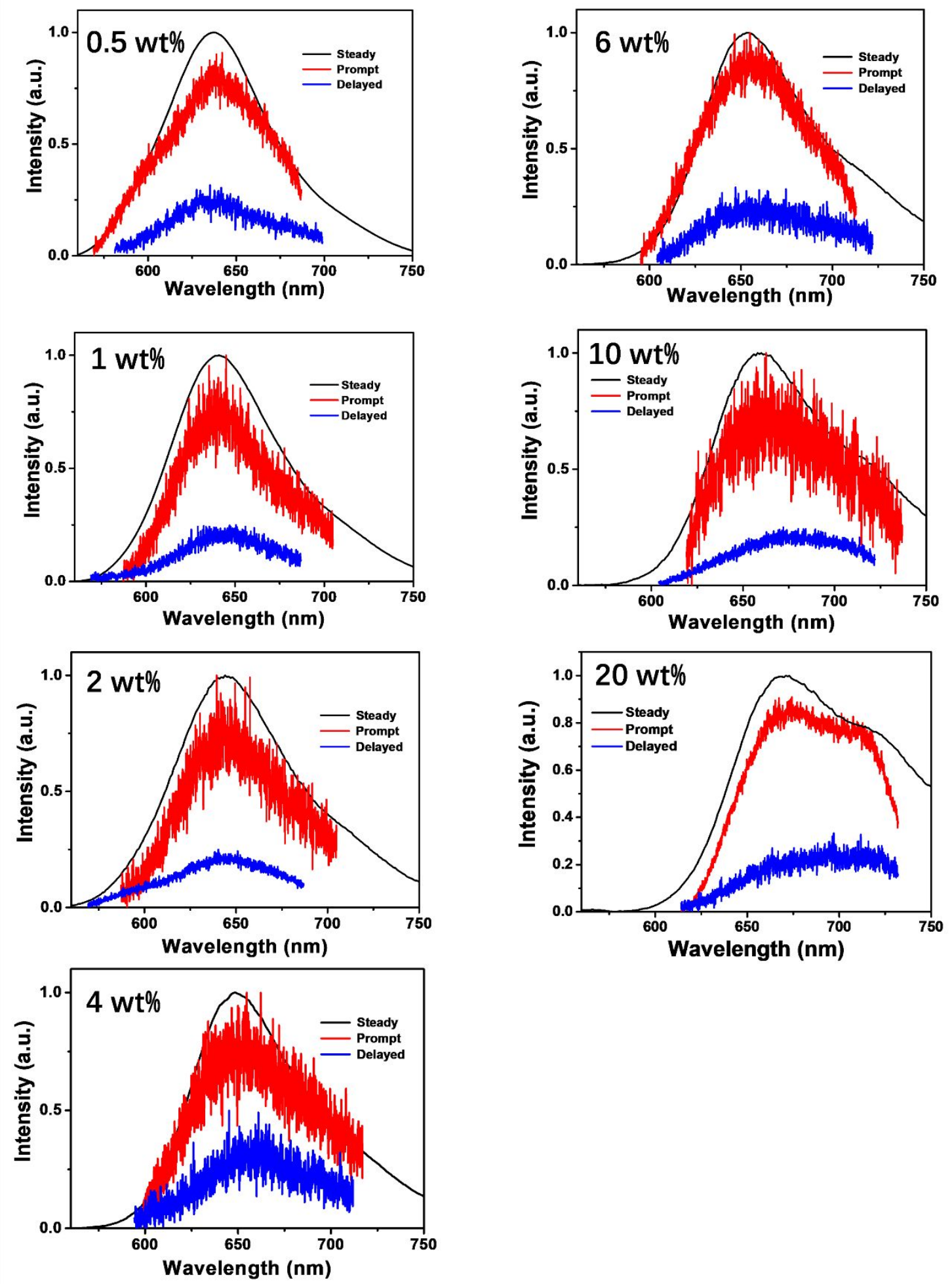

Figure S5. Steady-state PL (black lines), the prompt (blue lines), and delayed (red lines) spectra of CAZ-A/CBP MRs in different doping concentrations. 


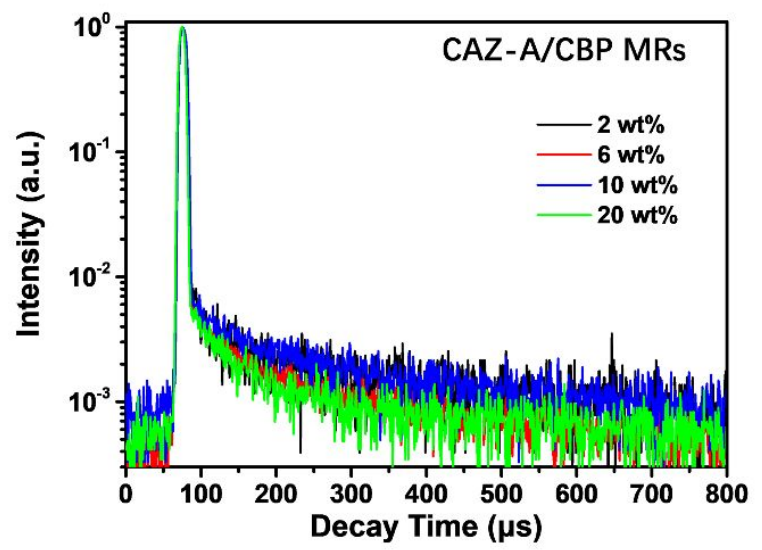

Figure S6. PL decay curves indicate similar TADF characteristics of CAZ-A/CBP MRs in different doping concentrations at room temperature. Photoluminescence was integrated over the spectral range of $600-700 \mathrm{~nm}$. 
a

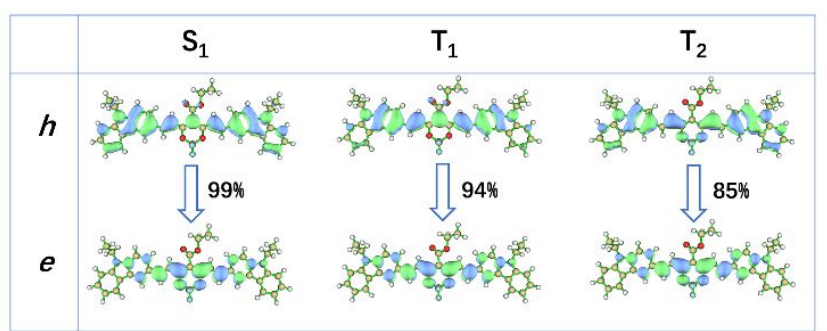

b

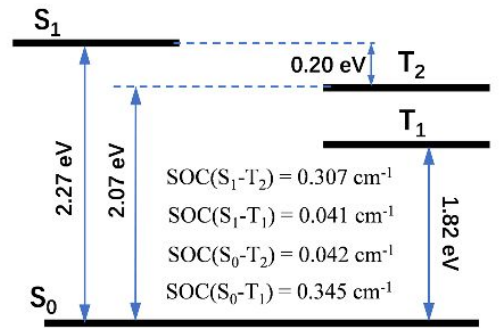

Figure S7. a) Nature transition orbitals (NTOs) (h: hole and e: electron) describing the $\mathrm{S}_{1}, \mathrm{~T}_{1}$ and $\mathrm{T}_{2}$ states of CAZ-A molecule. The isovalue is 0.025 . b) The theoretically calculated energy levels of $\mathrm{S}_{1}, \mathrm{~T}_{1}$ and $\mathrm{T}_{2}$ states and the spin-orbit coupling (SOC) between the energy states involved.
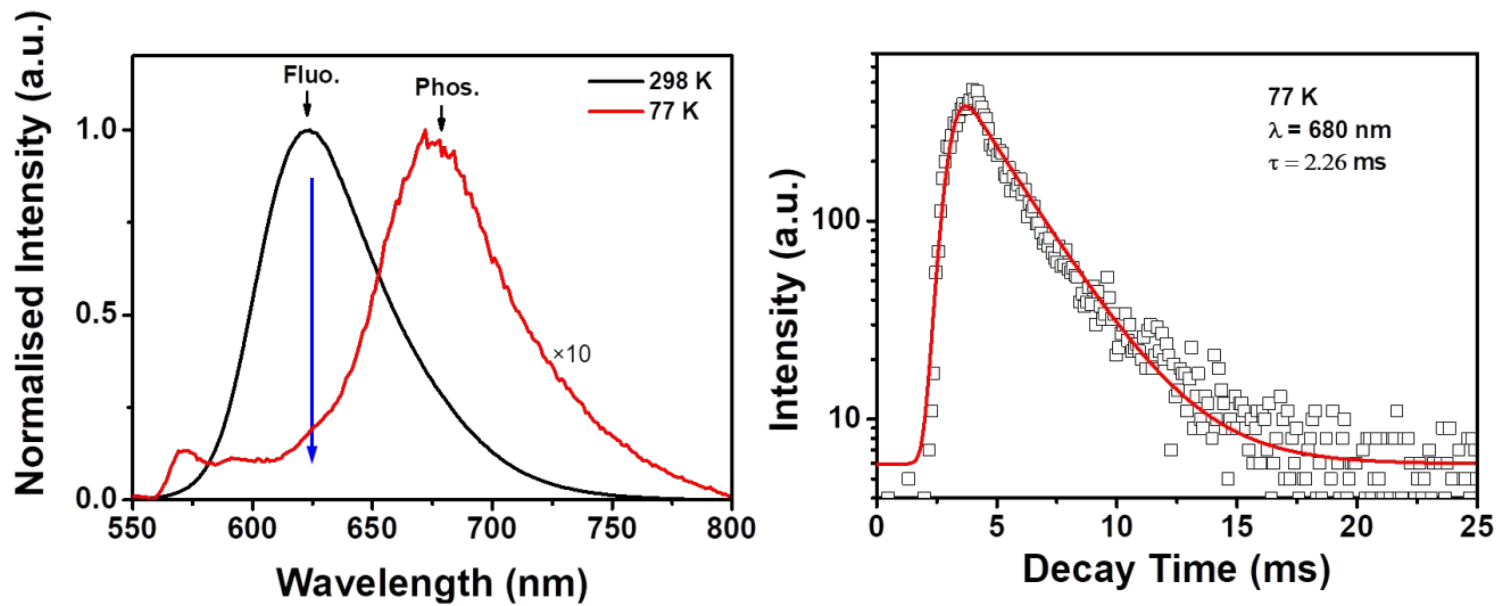

Figure S8. Left: Steady PL spectrum shows a fluorescent emission (back line, $298 \mathrm{~K}$ ) and a phosphorescent emission (red line, $77 \mathrm{~K}$ ) of CAZ-A dilute $\mathrm{CHCl}_{3}$ solution. Right: PL intensity at $680 \mathrm{~nm}$ versus the decay time shows a phosphorescence lifetime of $2.26 \mathrm{~ms}$ at $77 \mathrm{~K}$ in air. 
Note S3. Estimation of the energy difference between the $S_{1}$ and $T_{1}$ states $\left(\Delta E_{S T}\right)$ of the 4 wt\% MRs. The values are determined from PLQY and the lifetimes of the fluorescence and TADF components. $\Phi_{\mathrm{F}}$ and $\Phi_{\mathrm{DF}}$ are calculated by their ratios of the integral areas in the time-resolved spectrum in Figure S9. Both the prompt and delayed components exhibit biexponential decays, these can be attribute to the coexist of the monomer and dimer in the doping MRs, so $\tau_{\mathrm{F}}$ and $\tau_{\mathrm{DF}}$ are the average lifetime of the prompt and delayed components, they are calculated by $\tau_{\mathrm{av}}=\sum A_{\mathrm{i}} \tau_{\mathrm{i}}^{2} / \sum A_{\mathrm{i}} \tau_{\mathrm{i}}$, where $A_{\mathrm{i}}$ is the pre-exponential for lifetime $\tau_{\mathrm{i}}\left(A_{\mathrm{i}}\right.$ is the proportion of $\tau_{\mathrm{i}}$ here).

$$
\begin{array}{ll}
\Phi=0.17 & \\
\Phi_{\mathrm{F}}=0.149 & \\
\Phi_{\mathrm{DF}}=0.021 & \\
\tau_{\mathrm{F}}=1.27 \mathrm{~ns} & \\
\tau_{\mathrm{DF}}=191 \mu \mathrm{s} & \\
k_{\mathrm{F}}=1.17 \times 10^{8} \mathrm{~s}^{-1} & { }_{\mathrm{F}}=\Phi_{\mathrm{F}} / \tau_{\mathrm{F}} \\
k_{\mathrm{IC}}=5.71 \times 10^{8} \mathrm{~s}^{-1} & \Phi_{\mathrm{F}}=k_{\mathrm{F}} /\left(k_{\mathrm{F}}+k_{\mathrm{IC}}+k_{\mathrm{ISC}}\right) \\
k_{\mathrm{ISC}}=9.72 \times 10^{7} \mathrm{~s}^{-1} & \Phi_{\mathrm{IC}}=k_{\mathrm{IC}} /\left(k_{\mathrm{F}}+k_{\mathrm{IC}}+k_{\mathrm{ISC}}\right) \\
\Phi_{\mathrm{IC}}=0.73 & \Phi_{\mathrm{ISC}}=1-\Phi_{\mathrm{IC}}-\Phi_{\mathrm{F}}=k_{\mathrm{ISC}} /\left(k_{\mathrm{F}}+k_{\mathrm{IC}}+k_{\mathrm{ISC}}\right) \\
\Phi_{\mathrm{ISC}}=0.10 & k_{\mathrm{DF}}=\Phi_{\mathrm{DF}} / \Phi_{\mathrm{ISC}} \tau_{\mathrm{DF}} \\
k_{\mathrm{DF}}=1.10 \times 10^{3} \mathrm{~s}^{-1} & k_{D F}=\frac{1}{3}\left(k_{F} e x p \frac{-\Delta E_{S T}}{R T}\right) \\
\Delta E_{\mathrm{ST}}=0.26 \mathrm{eV} & k_{\mathrm{RISC}}=k_{\mathrm{F}} k_{\mathrm{DF}} /\left(k_{\mathrm{F}}-k_{\mathrm{ISC}}\right) \\
k_{\mathrm{RISC}}=6.5 \times 10^{3} \mathrm{~s}^{-1} &
\end{array}
$$



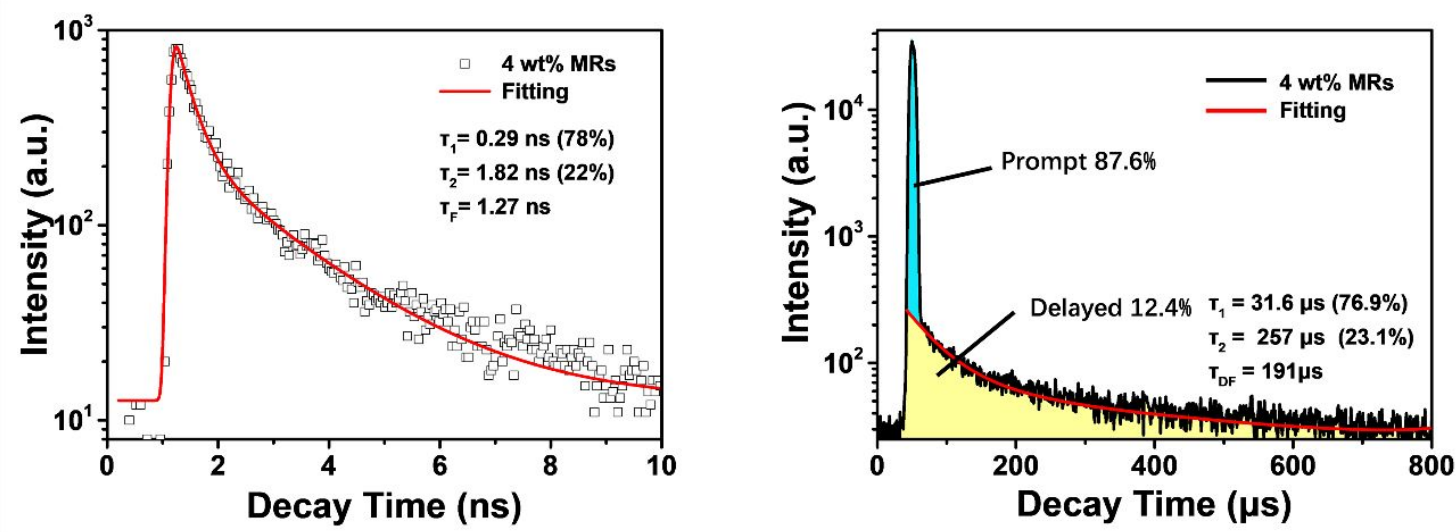

Figure S9. Transient decay spectra of $4 \mathrm{wt} \% \mathrm{CAZ}-\mathrm{A} / \mathrm{CBP}$ MRs at $298 \mathrm{~K}$. 
a

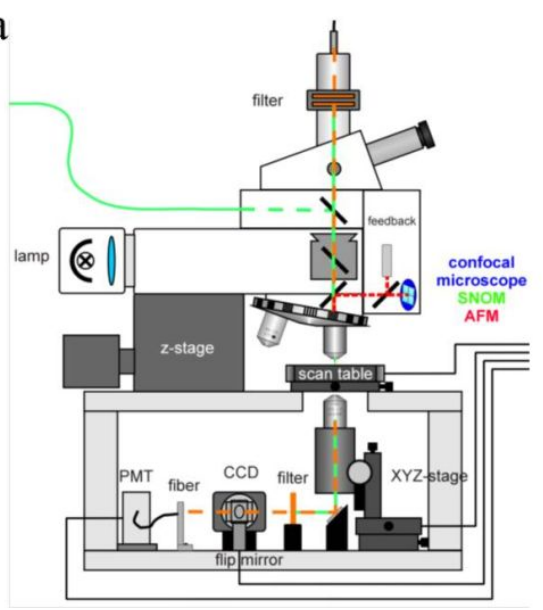

b

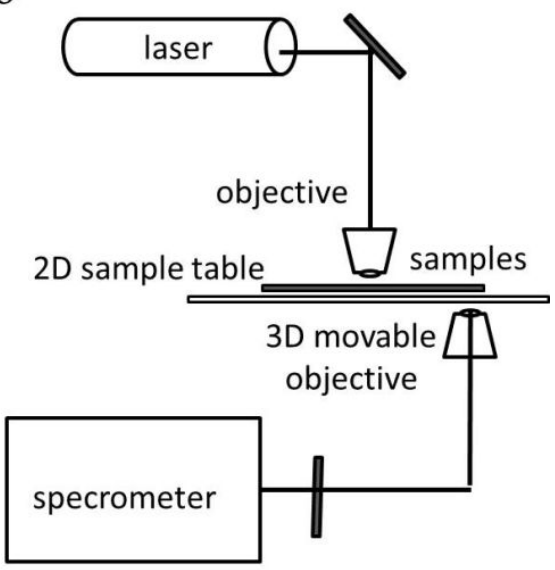

Figure S10. Schematic demonstration of the experimental setup for the optical characterization: a) the confocal optical microscopy and b) the transmittance optical path for the waveguide measurements.

Single CAZ-A/CBP MR was investigated at room temperature in air by a home-made optical microscopy equipped with a $50 \times 0.9$ NA excitation objective. The samples were placed on the 2D-movavble sample table of the optical microscope. The second harmonic (400 nm, $150 \mathrm{fs}, 1$ $\mathrm{kHz}$ ) of a regenerative amplifier (Spitfire, Spectra Physics) seeded with a mode-locked Ti:sapphire laser (Tsunami, Spectra Physics) was focused to a 2- $\mu \mathrm{m}$-diameter spot to excite the selected CAZ-A/CBP MR. The pump intensity of the pulse laser varied using a series of metallic neutral density filters. Then PL spectra were collected underneath by using a same $50 \times 0.9$ NA objective. A 430-nm long-wave pass dielectric filter was used to block any scattered excitation light. Finally the collected PL was coupled to an optical fiber and detected using a liquid-nitrogen-cooled CCD (SPEC-10-400B/LbN, Roper Scientific) attached to a polychromator (Spectropro-550i, Acton Research Corp.) 

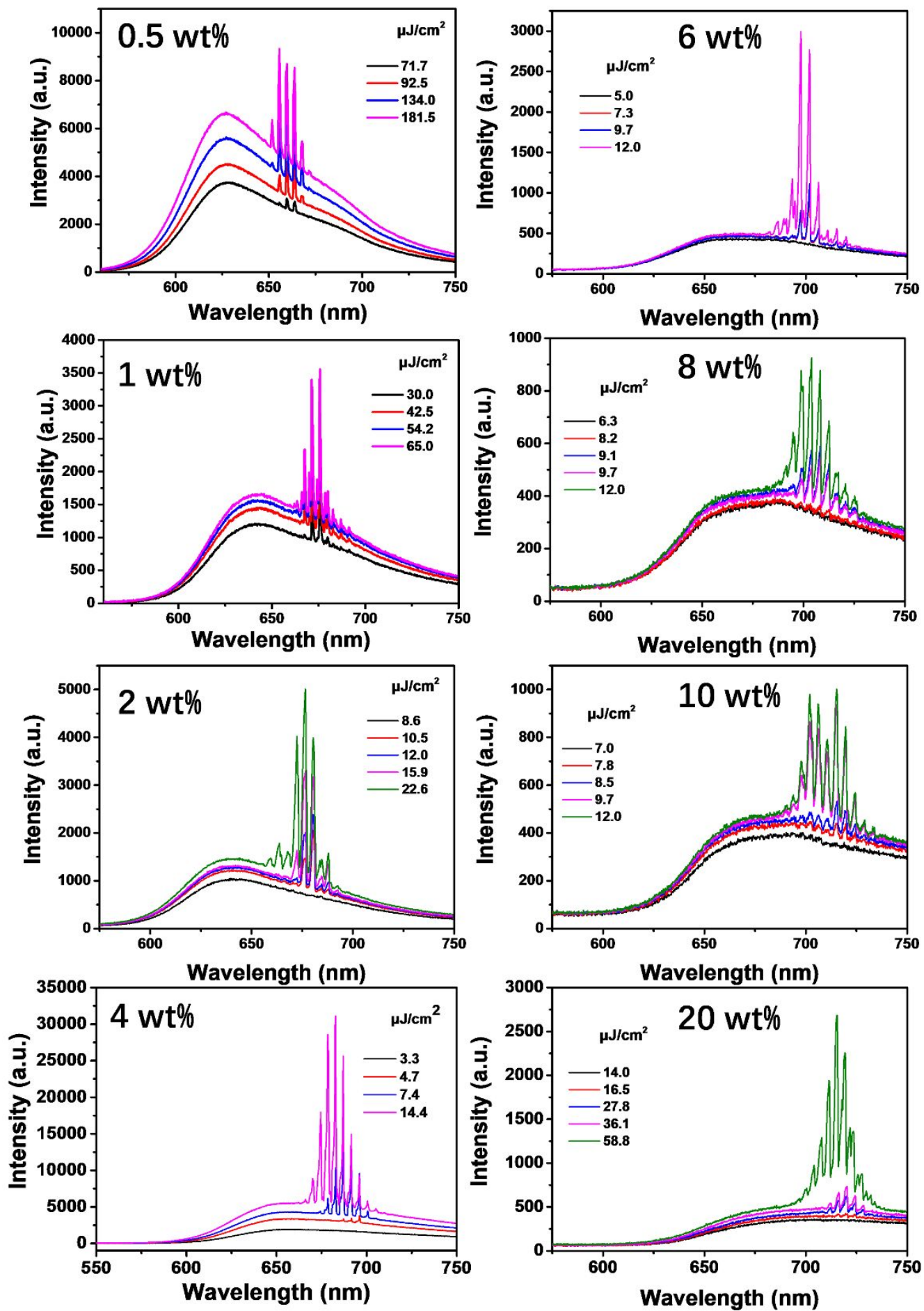

Figure S11. The laser spectra of an individual MR $(\mathrm{D}=19 \mu \mathrm{m})$ at gradual increasing doping concentrations shows a red-shift from nearly $650 \mathrm{~nm}$ to $725 \mathrm{~nm}$. 

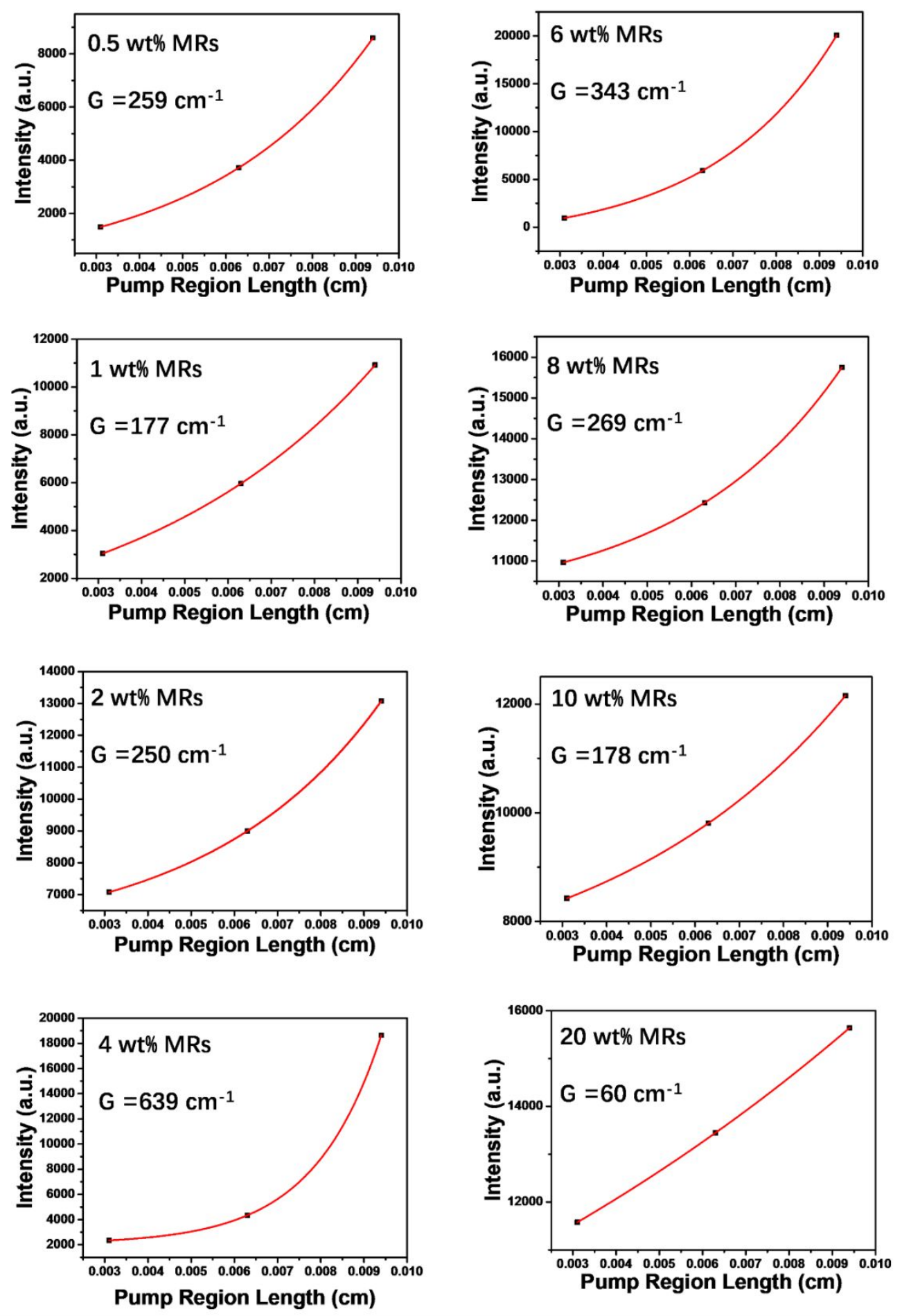

Figure S12. Laser gain curves for CAZ-A/CBP MRs with different doping concentrations at pump intensities above their thresholds. 
a

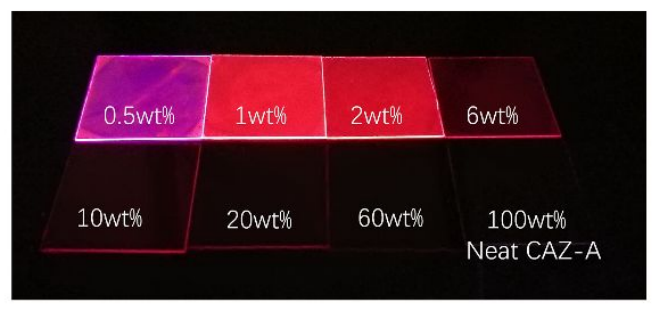

b $\widehat{i}$

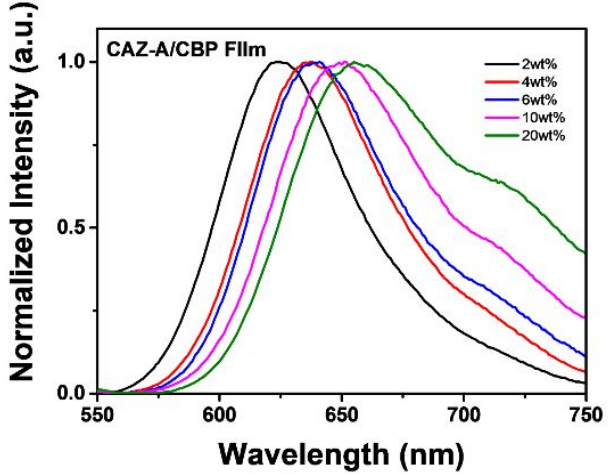

Figure S13. Photographs under UV light (a) and Steady PL spectra (b) of CAZ-A/CBP films at various doping concentrations (each sample: $2 \times 2 \mathrm{~cm} 2$ ).

CAZ-A/CBP films were produced by spin coating. A mixture of different concentrations of the solution was evenly applied to the glass substrate, and the homomixer was set to $2000 \mathrm{r} / \mathrm{min}$ for 1 min to get the CAZ-A/CBP doped films of different doping concentrations. The thickness of these different doped films is between 800 and $900 \mathrm{~nm}$.
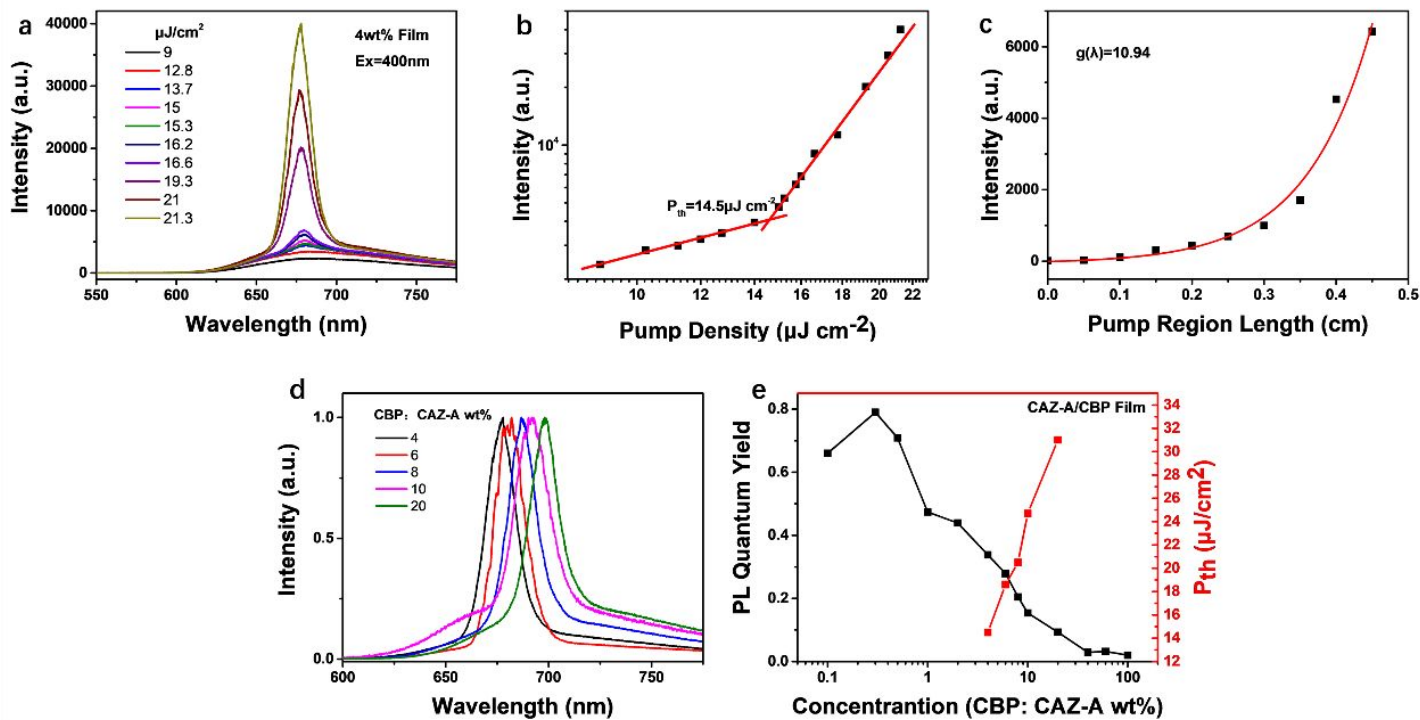

Figure S14. Amplified spontaneous emissions (ASE) and their gains in CAZ-A/CBP films with different doping concentrations. a) Emission spectra measured from the edge of a CAZ-A/CBP thin film at $4 \mathrm{wt} \%$ concentration at different excitation densities. b) Output intensity from the edge emission versus excitation density. c) The dot plot of the PL intensity (black) and its 
simulated curve (red) versus the linear excitation spot widths yielded an ASE gain factor of G $=10.94 \mathrm{~cm}^{-1}$ in $4 \mathrm{wt} \%$ CAZ-A/CBP film. b) shows a red shift from $650 \mathrm{~nm}$ to $700 \mathrm{~nm}$ of the peak wavelengths of the ASEs from the edges of the CAZ-A/CBP thin films at various doping concentrations. e) Dotted line diagram of luminescence quantum yield versus doping ratio (black) and ASE threshold versus doping ratio (red). Although the $0.3 \mathrm{wt} \%$ film has the highest PLQY (78\%), the ASE phenomenon begins to be obtained when the doping concentration is 4 $\mathrm{wt} \%$, which should have the same interpretation as MRs. As the doping concentration increases to $20 \mathrm{wt} \%$, the laser threshold rises from $14.5 \mu \mathrm{J} / \mathrm{cm}^{2}$ to $31 \mu \mathrm{J} / \mathrm{cm}^{2}$. As compared with the corresponding concentrations of MRs, films obviously exhibit higher thresholds, which is further proof that our MR structures have better optical confinement capability and greatly improves the optical gain of CAZ-A. 